\title{
ENSAYO
}

\section{Estándares de calidad para un servicio dietético de leche, en un hospital de niños con enfermedades crónicas}

\section{Quality standards for a dietary milk service in a hospital for children with chronic diseases}

\begin{abstract}
Pediatric patients with chronic diseases and long term hospitalizations are at high risk to contract food infections affecting their conditions and increasing their risk of mortality. A food quality control and safety system such the Hazard Analysis Critical Control units Points (HACCP) could reduce this risk to the lowest allowing to identify the points of actions and the preventives measures to avoid the risk of microbial growth. The present article review the recommendations and norms that are possible and available for the preparation of the infant powder formula, as well as, describing the formulas that are provide in the children hospital, to patients with chronic diseases, with the purpose of guaranty safety in the production system, so its final outcome is adequate and innocuous in its composition.
\end{abstract}

Key words: Enteral feeding., food safety., HACCP., infant powder formula.
Edson Bustos A. (1)

Yasna Franulic P. (2)

Natalia Farías Z. (2)

(1) Hospital Josefina Martínez. Carrera de Nutrición y Dietética, Pontificia Universidad Católica de Chile, Santiago, Chile. (2) Hospital Josefina Martínez, Santiago, Chile.

Dirigir la correspondencia a:

Edson Bustos Arriagada e-mail: edsonbustos@gmail.com

Este trabajo fue recibido el 2 de Julio de 2015 y aceptado para ser publicado el 25 de Octubre de 2015.

\section{INTRODUCCIÓN}

La lactancia materna $(L M)$ es la alimentación más completa para el lactante, tanto en seguridad como calidad; otorga suficiencia de nutrientes, ayuda a prevenir infecciones, favorece el desarrollo global y disminuye la morbimortalidad infantil $(1,2)$. La OMS y UNICEF recomiendan la LM exclusiva al menos los primeros seis meses de vida, complementada posteriormente con otros alimentos hasta los dos años o más (3-5).

Los niños con enfermedades crónicas presentan muchas dificultades para mantener la LM, más aún si son hospitalizados con frecuencia o de modo prolongado. Entre las causas que explican dicha dificultad se encuentra su insuficiencia, la imposibilidad de amamantarlos y/o extracción inadecuada. En dichas situaciones se recurre a fórmulas lácteas infantiles en polvo, en forma exclusiva o complementaria. Con frecuencia la vía enteral es necesaria de modo temporal o permanente (6).

Sin embargo, las fórmulas no son estériles y por ende es factible el desarrollo microbiano, siendo una posible fuente de infecciones $(7,8)$. En los hospitales, las técnicas inadecuadas de fabricación, recepción, almacenamiento, preparación y distribución de las fórmulas enterales constituyen un riesgo importante de infecciones asociadas a la atención de salud (IAAS) $(9,10)$. El Sistema de Análisis de Peligros y de Puntos Críticos de Control (HACCP) aceptado por la Comisión del Codex Alimentarius (CCA), permite identificar los riesgos específicos y medidas de control, con el objetivo de garantizar la inocuidad y calidad de los alimentos $(10,11)$.

El Servicio Dietético de Leche (SEDILE), es un espacio físico diseñado específicamente para la elaboración de fórmulas lácteas infantiles que cubran los requerimientos nutricionales de cada paciente (12). Es una unidad fundamental dentro de la estructura de un centro hospitalario pediátrico, donde se realiza la recepción y almacenamiento de las materias primas, para después preparar, esterilizar y distribuir las fórmulas lácteas e hidrataciones (13).

El hospital Josefina Martínez es un hospital de transición y larga estadía para niños con enfermedades respiratorias crónicas (14) muchas de ellas asociadas a patologías neurológicas, neuromusculares y/o derivadas de la 
prematurez $(15,16)$. Estos pacientes tienen alto riesgo de desnutrición, constipación y déficit nutricionales específicos tales como hierro y vitamina D (17), por lo cual el manejo nutricional es fundamental.

En niños hospitalizados por enfermedades crónicas, la prevalencia de desnutrición es 25 a 30\%, siendo la causa más frecuente la del sistema respiratorio (18-20). En nuestro hospital esta prevalencia es cercana al 14\%, y justifica el seguimiento regular para entregar un adecuado y seguro apoyo nutricional, sobre todo considerando que es administrado por vía enteral a $80 \%$ de los pacientes.

Motivados por esta realidad, el objetivo de este estudio fue realizar una revisión bibliográfica del tema, para mostrar una información actualizada sobre los estándares de calidad para un servicio dietético de leche, en un hospital de niños con enfermedades crónicas.

\section{METODOLOGÍA}

Se realizó una búsqueda en la base de datos Medline/ Pubmed y las palabras claves utilizadas fueron: powdered infant formulas, HACCP milk, HACCP hospital, HACCP and enteral feeding. En la base de datos Scielo, sitio web del Codex Alimentarius Chile, FAO y de la OMS, las palabras claves utilizadas fueron: Servicio Dietético de Leche, formulas enterales infantiles y lactancia materna. La búsqueda se realizó durante los meses de Marzo a Junio de 2015.

Se encontraron 103 artículos de los cuales se seleccionaron sólo las investigaciones y normas nacionales e internacionales realizadas en los últimos 15 años, que abordan los temas de calidad y funcionamiento de SEDILE intrahospitalario, en los idiomas español e inglés. Después de aplicar estos criterios se seleccionaron 33 artículos, trece de ellos versaban sobre estándares de calidad en la fabricación de fórmulas lácteas en polvo, siete sobre desnutrición infantil intrahospitalaria, seis de microorganismos contaminantes de fórmulas lácteas infantiles en polvo, cinco sobre lactancia materna y alimentación normal del niño menor de 2 años y otros tres de los cuales uno corresponde a la reseña histórica del Hospital Josefina Martínez, otro a las orientaciones técnicas de Chile para la implementación de un SEDILE y el tercero al Reglamento Sanitario de los Alimentos en Chile.

\section{RESULTADOS}

Los procedimientos de aseguramiento de calidad planificados para el SEDILE del Hospital Josefina Martínez contemplan las sugerencias establecidas por la OMS/FAO correspondientes al cumplimiento de las Buenas Prácticas de Higiene (BPH) tanto en la fabricación de las fórmulas en polvo como durante su reconstitución (11), estas indicaciones están destinadas a los siguientes productos:

- Preparaciones destinadas a lactantes y de uso médico especial que sirven como única fuente de nutrición.

- Preparaciones de continuación de uso conjunto a la alimentación complementaria a partir del sexto mes de vida.

- $\quad$ Preparados para fines médicos especiales que son empleados como sustitutos o complementos de la leche materna.

- $\quad$ Fortificantes de leche materna.

Estas preparaciones se diferencian de los líquidos listos para el consumo (ready to hang o RTH) por el proceso de esterilización a los cuales estos últimos son sometidos, es por esto que han sido incorporados a los centros hospitalarios de manera creciente por su seguridad, aunque su costo primario es mayor (11).

Los preparados en polvo no son estériles y en Chile el Reglamento Sanitario de los Alimentos, indica las cantidades mínimas y máximas de microorganismos que pueden estar presentes en estos preparados (tablas 1, 2 y 3) (21).

Su inocuidad microbiológica está vinculada al cumplimiento estricto de buenas prácticas de higiene durante su fabricación o uso posterior. Luego de examinar la información disponible, la reunión de expertos de la FAO/OMS sobre la inocuidad microbiológica de las preparaciones en polvo para lactantes, concluyeron que la contaminación intrínseca de estos preparados para niños estaba dada con clara causalidad por Cronobacter sakazakii y Salmonella. En la tabla 4 se describen las tres categorías de microorganismos allí consideradas, en cuanto a la relación causal entre su presencia en los preparados y ocurrencia de enfermedad en los lactantes $(22,23)$.

A continuación se describe brevemente las principales características de los dos principales microorganismos:

\section{TABLA 1}

Fórmulas deshidratadas para niños menores de 12 meses.

\begin{tabular}{|c|c|c|c|c|c|c|}
\hline \multirow[b]{2}{*}{ Parámetro } & \multicolumn{2}{|c|}{ Plan de muestreo } & \multicolumn{4}{|c|}{ Límite por gramo } \\
\hline & Categoría & Clase & $\mathrm{n}$ & $\mathrm{C}$ & $\mathrm{m}$ & $M$ \\
\hline Rcto. Aerobios Mesóf. (*) & 5 & 3 & 5 & 2 & $10^{3}$ & 104 \\
\hline Rcto. Aerobios Mesóf. $\left({ }^{*}\right)\left({ }^{* *}\right)$ & 5 & 3 & 5 & 2 & 104 & $5 \times 104$ \\
\hline Coliformes & 6 & 3 & 5 & 1 & $<3$ & 20 \\
\hline E. coli & 10 & 2 & 5 & 0 & $<3$ & - \\
\hline B. cereus & 8 & 3 & 5 & 1 & 102 & $10^{3}$ \\
\hline C. perfringens $\left(*^{* *}\right)$ & 8 & 3 & 5 & 1 & 102 & $10^{3}$ \\
\hline S. aureus & 8 & 3 & 5 & 1 & 10 & 102 \\
\hline Salmonella en $25 \mathrm{~g}$ & 11 & 2 & 10 & 0 & - & - \\
\hline
\end{tabular}


Cronobacter sakazakii: Es una bacteria Gram-negativa móvil, no formadora de esporas, anaerobia facultativa $(25,26)$. La FAO/OMS determinó que los lactantes menores, neonatos, prematuros (26), lactantes de bajo peso al nacer, inmunodeprimidos e hijos de madres portadoras de VIH $(22,23)$ son los grupos de mayor riesgo de contraer la infección por este patógeno.

La incidencia de infección es baja, pero con graves consecuencias. Las manifestaciones más comunes son meningitis y bacteremia. Estas infecciones se han presentado tanto en pacientes hospitalizados como ambulatorios (22).

En Chile, el RSA no dispone de normas para el manejo e identificación microbiológica del Cronobacter sakazakii (21) en fórmulas de prematuros (27), inicio o continuación. Esta situación hace necesario un mayor énfasis en la investigación epidemiológica y búsqueda clínica de esta bacteria.

Salmonella: Es una bacteria Gram negativa, anaeróbica facultativa de la familia Enterobacteriaceae. Los síntomas se caracterizan por fiebre alta, dolor abdominal, diarrea, náusea y a veces vómitos (28).

Principal patógeno asociado a transmisión alimentaria. La incidencia de salmonelosis en lactantes es 8 veces superior que en otras edades, correspondiendo este grupo al de mayor riesgo de morbi-mortalidad (23).

Las bacterias C. sakazakii y Salmonella pueden introducirse en las preparaciones en base a fórmulas lácteas o entérales en polvo durante cuatro etapas del proceso:

1. Durante la fabricación, en las operaciones de mezcla de los ingredientes en seco.

2. Cuando la preparación es realizada en un ambiente contaminado.

3. Cuando el preparado en polvo es abierto.

4. Durante la reconstitución del preparado efectuado por el personal de alimentación (23).

Las medidas preventivas adoptadas en nuestro SEDILE, están dirigidas a minimizar el riesgo de contaminación en los procesos 3 y 4 principalmente. Para asegurar la calidad e inocuidad alimentaria se aplica el sistema HACCP que tiene distintos niveles de acción, dentro de los cuales destacan:

1. Realizar análisis de peligros.

2. Evaluar los Puntos Críticos de Control (PCC).

3. Establecer límites críticos.

4. Disponer sistemas de vigilancia de control de los PCC.

5. Implementar medidas correctivas cuando la vigilancia indica que un PCC no está controlado.

6. Realizar procedimientos de documentación y comprobación del funcionamiento de $\operatorname{HACCP}(29,30)$.

Dentro de las etapas para la elaboración de fórmulas lácteas implementadas en nuestro hospital (figura 1), las que tienen incidencia directa en la inocuidad, calidad y control de los puntos críticos son $(29,31)$ :

a) Remodelación con unidireccionalidad de la planta física y autonomía en el recurso humano y financiero.

b) Selección de materias primas y envases: proveedores certificados, recepción de productos y verificación visual de estado físico.

\section{TABLA 2}

Fórmulas deshidratadas para niños mayores de 12 meses.

\begin{tabular}{|c|c|c|c|c|c|c|}
\hline \multirow[b]{2}{*}{ Parámetro } & \multicolumn{2}{|c|}{ Plan de muestreo } & \multicolumn{4}{|c|}{ Límite por gramo } \\
\hline & Categoría & Clase & $\mathrm{n}$ & $c$ & $\mathrm{~m}$ & $M$ \\
\hline Rcto. Aerobios Mesóf. $\left({ }^{*}\right)$ & 5 & 3 & 5 & 2 & 104 & $5 \times 104$ \\
\hline Coliformes & 6 & 3 & 5 & 1 & & 20 \\
\hline E. coli & 10 & 2 & 5 & 0 & $<3$ & - \\
\hline B. cereus & 8 & 3 & 5 & 1 & $<3$ & $10^{3}$ \\
\hline S. aureus & 8 & 3 & 5 & 1 & 102 & 102 \\
\hline Salmonella en $25 \mathrm{~g}$ & 11 & 2 & 10 & 0 & 10 & - \\
\hline
\end{tabular}

(*) Excepto para fórmulas con cultivos bacterianos

Fuente: Reglamento Sanitario de los alimentos (RSA) 2015. Título V, articulo 173.

\section{TABLA 3}

Preparaciones reconstituidas, listas para el consumo administradas exclusivamente por biberones o mamaderas.

\begin{tabular}{|c|c|c|c|c|c|c|}
\hline \multirow[b]{2}{*}{ Parámetro } & \multicolumn{2}{|c|}{ Plan de muestreo } & \multicolumn{4}{|c|}{ Límite por gramo } \\
\hline & Categoría & Clase & $\mathrm{n}$ & C & $\mathrm{m}$ & $M$ \\
\hline Rcto. Aerobios Mesóf. & 6 & 3 & 5 & 1 & 102 & 102 \\
\hline E. coli & 10 & 2 & 5 & 0 & $<3$ & - \\
\hline B. cereus & 10 & 2 & 5 & 0 & $<10$ & - \\
\hline S. aureus & 10 & 2 & 5 & 0 & $<10$ & - \\
\hline Salmonella en $25 \mathrm{~g}$ & 10 & 2 & 5 & 0 & - & - \\
\hline
\end{tabular}


c) Sanitización y esterilización inicial de mamaderas y utensilios, son considerados PCC, ya que determina el riesgo de contener microorganismos. Se incluye utilización de productos químicos certificados para sanitización, y esterilización a través de un equipo de autoclave de vapor húmedo.

d) Elaboración, envasado y rotulación: la reconstitución de las fórmulas lácteas en polvo, proceso importante para evitar la proliferación de microorganismos, este PCC contempla medidas preventivas en higiene, sanitización de superficies y ambiente, temperatura de sala de preparado no superior a los $20^{\circ} \mathrm{C}$, vigilada a través de termómetros estables de pared, uso de agua potable hervida a temperatura $>70^{\circ} \mathrm{C}$, esta medición se realiza a través de termómetros móviles certificados. El personal debe ejecutar un adecuado lavado de manos (quirúrgico), uso de mascarillas y uniforme estéril. El envasado se realiza bajo las mismas condiciones de asepsia del personal, ambiente y superficies mencionadas anteriormente. El proceso continúa con el rotulado, donde se incluye la información del tipo de fórmula láctea, nombres y apellidos de paciente, fecha de elaboración, volumen total, horario de la alimentación y sala de hospitalización.

e) Refrigeración: PCC, por el riesgo de proliferación de microorganismos patógenos. La temperatura ideal es entre 0 y $5^{\circ} \mathrm{C}(32)$, el límite crítico establecido son $8^{\circ} \mathrm{C}$ y el tiempo de refrigeración no excede las 24 horas a temperatura óptima.

f) Transporte y distribución: a través de carro transportador refrigerado o de ambiente fresco, y sometidas al proceso de retermalización o "baño maría" a temperatura de $35^{\circ} \mathrm{C}$ durante 15 minutos.

g) Lavado y sanitización de material sucio: utilización de agua potable, productos químicos certificados y utensilios (hisopo).

h) Eliminación de productos desechables: se realiza en lugares del recinto hospitalario destinados para este fin, los cuales son seguros y están alejados del área de SEDILE.

Como método de verificación de inocuidad, es necesario el análisis microbiológico sistemático de las preparaciones lácteas, esto permite tener un control y registro de la actividad microbiana. Este análisis se realiza de manera trimestral a utensilios dispuestos para la preparación, mamaderas vacías, mamaderas recién preparadas y a las 24 horas de refrigeración. Los análisis se realizan para todos los microorganismos aeróbicos referenciados en el RSA, haciendo hincapié en aquellos con clara y posible causalidad (31), a excepción del C. sakasakii el cual no es analizado en los laboratorios de uso habitual.

Las medidas correctivas ante resultados microbiológicos positivos, son:

a) Verificación de posibles casos de infección alimentaria en niños que hayan consumido fórmulas lácteas afectadas y notificación al departamento de infecciones asociadas a la atención de salud (IAAS) del hospital.

b) Aviso al fabricante de fórmulas lácteas en polvo y no usar de lotes afectados.

c) Tomar nuevos muestreos microbiológicos a lotes afectados y a otros del mismo fabricante.

d) Revisión de todos los procesos de calidad e inocuidad en la preparación de fórmulas lácteas.

e) Revisión de las medidas preventivas asociadas a cada PCC (33).

\section{CONCLUSIÓN}

Son primordiales para la calidad, seguridad e inocuidad alimentaria asociada a los procesos de elaboración de preparaciones lácteas para pacientes pediátricos con enfermedades crónicas. Ello contribuye a una adecuada nutrición y evita que la alimentación sea un factor de riesgo que aumente la morbi-mortalidad y estadía hospitalaria. La aplicación de estas medidas de aseguramiento de calidad para la producción de fórmulas lácteas infantiles contribuye a disminuir y controlar los riesgos en todas las etapas de la preparación.

TABLA 4

Categorización de los microorganismos según causalidad de enfermedad a lactantes.

Clara causalidad

Cronobacter sakazakii (24)

Salmonella entérica

Posible causalidad

Enterobacter aglomeranss

Enterobacter cloacae

Klebsiella oxytoca

Klebsiella pneumoniae

Citrobacter koseri

Citrobacter freundii

Hafnia alvei

\author{
Causalidad \\ menos probable
}

Bacillus cereus
Clostridium botulinum

Clostridium difficile

Clostridium perfringens

Listeria monocytogenes

Staphylococcus aureus
Causan enfermedad en el lactante y preescolares, y se ha demostrado que los preparados lácteos fueron el vehículo o fuente de infección.

Causan enfermedad en lactante y preescolares, pero no se ha demostrado que el vehículo o fuente de infección sean estos preparados lácteos.

Causan enfermedad en lactantes y preescolares, pero no han sido identificados en las preparaciones lácteas o incluso al serlo, no se ha demostrado su relación con la enfermedad. 


\section{RESUMEN}

Los pacientes pediátricos con enfermedades crónicas y hospitalizaciones prolongadas tienen un alto riesgo de contraer infecciones de origen alimentario, lo que aumenta su morbilidad y mortalidad. El aseguramiento de la calidad y los sistemas de inocuidad de los alimentos puede reducir al mínimo este riesgo, entre ellos, el Sistema de Análisis de Peligros y Puntos Críticos de Control (HACCP: Hazard Analysis Critical Control Points) permite identificar los puntos de acción y las medidas preventivas para evitar el riesgo de crecimiento microbiano. Este artículo revisa las recomendaciones y normas disponibles para la preparación de fórmulas lácteas en base a polvo, y describe aquellas que se aplican en un hospital de niños con enfermedades crónicas para garantizar la seguridad de todo el proceso y que el producto final sea adecuado e inocuo en su composición.

Palabras clave: SEDILE, alimentación enteral, HACCP, inocuidad alimentaria, fórmulas infantiles en polvo.

\section{BIBLIOGRAFÍA}

1. Jarpa C. Cerda J. Terrazas C. Cano C. Lactancia materna como factor protector de sobrepeso y obesidad en preescolares, Rev Chil Pediatr 2015; 86(1):32-7.

2. Paz R. Zalles L. Cruz W. Lactancia Materna vs Nuevas Fórmulas Lácteas Artificiales: Evaluación del Impacto en el Desarrollo, Inmunidad, Composición Corporal en el Par Madre/Niño, Gac Med Bol. 2011; 34 (1): 6-10.

3. Kramer MS. Kakuma R. Optimal duration of exclusive breastfeeding, Cochrane Database Syst Rev 2012; doi: 10.1002/14651858.CD003517.pub2.

4. OMS (2003). Estrategia mundial para la alimentación del lactante y niño pequeño, OMS Ginebra 2003, p. 8-10. (acceso: http://whqlibdoc.who.int/publications/2003/9243562215.pdf?ua=1, última visita 02/06/15)

5. Castillo C. Balboa P. Torrejón C. Bascuñan K. Uauy R. Alimentación normal del niño menor a 2 años, Rev Chil

\section{FIGURA 1}

Flujograma de elaboración y distribución de fórmulas lácteas infantiles en polvo en el SEDILE.

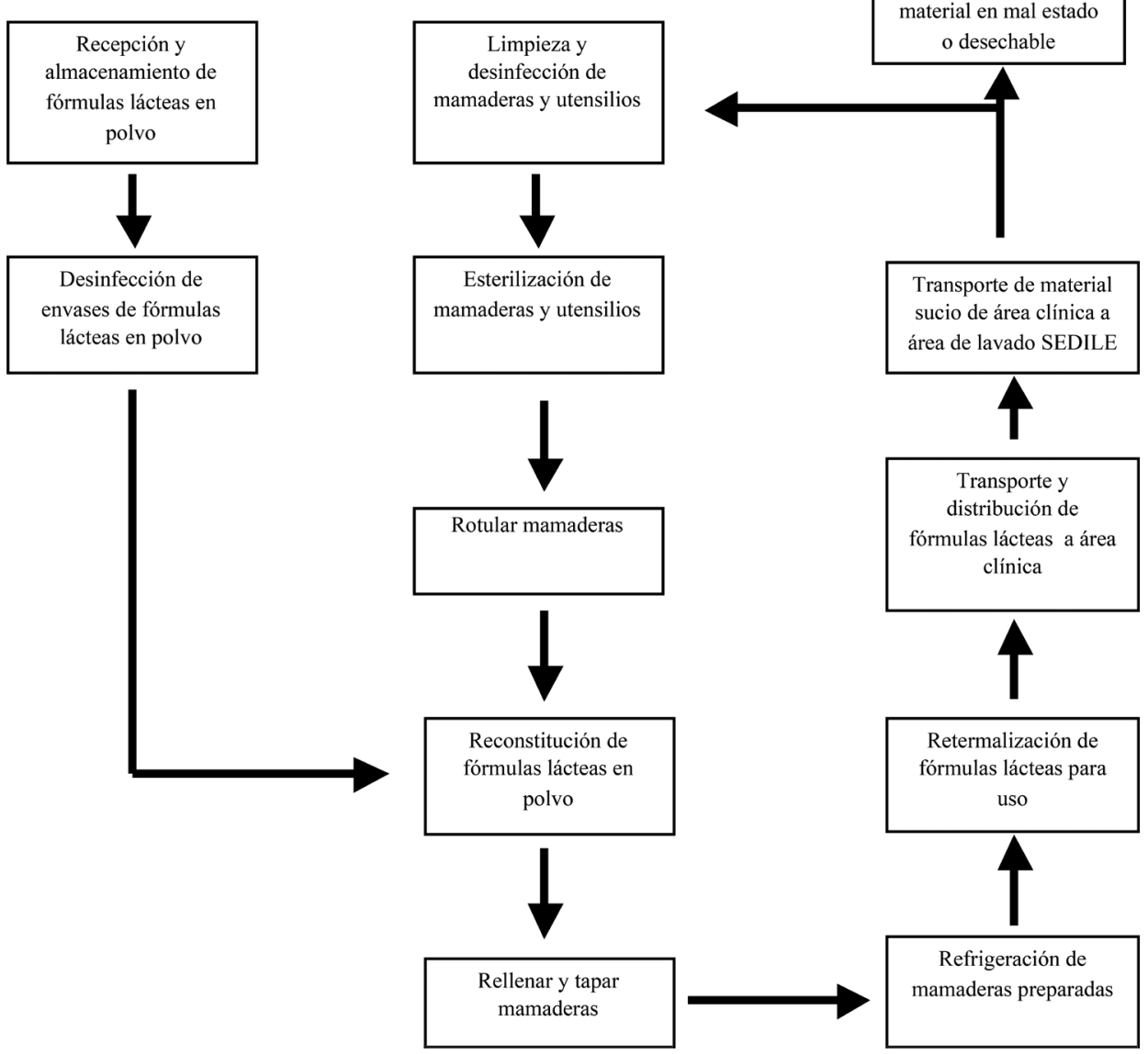


Pediatr. 2013; 84 (5): 565-72.

6. Mehta NM. Compher C. A.S.P.E.N. clinical guidelines: nutrition support of the critically ill child, Parenter Enteral Nutr. 2009; 33:260-76.

7. Kim H. Ryu J. Beuchat R. Attachment of and Biofilm Formation by Enterobacter Sakazakii on Stainless and Enteral Feeding Tubes, Appl Environ Microbiol. 2006; 72: 5846-56.

8. Lara S. Domecq C. Atalah E. Evaluación de buenas prácticas de manufactura en la elaboración de fórmulas enterales en hospitales públicos de Santiago, Chile, Nutr Hosp. 2013; 28(6):2021-2.

9. FAO/WHO (2006). Enterobacter sakazakii and Salmonella in powdered infant formula: Meeting report. Second Risk Assessment Workshop. 16-20th January, Rome, Italy 2006, p. 15-60. (acceso: ftp://ftp.fao.org/ ag/agn/jemra/e_sakakazii_salmonella.pdf, Última visita 02/06/15).

10. FAO/OMS (1997). Sistema de análisis de peligros y de puntos críticos de control (HACCP) y directrices para su aplicación. Anexo al CAC/RCP-1 (1969), Rev. 3 (1997). (acceso: http://www.fao.org/docrep/005/y1579s/ y1579s03.htm , Última visita 02/06/15)

11. FAO/OMS (2008). Programa conjunto FAO/OMS sobre las normas alimentarias. Comisión del Codex Alimentarius. $31^{\circ}$ periodo de sesiones Ginebra (Suiza), 30 de junio - 4 de julio 2008. (acceso: www.codexalimentarius.org/download/report/686/al31_13s.pdf ,Última visita 02/06/15).

12. MINSAL (2012). Orientación Técnica para Servicios Dietéticos de Leche (Sedile) y Central de Fórmulas Enterales (Cefe).

13. Mestre G. Masuda C. Brea M. Levy L. Pico M. Blasi S. Evaluación del riesgo de infecciones alimentarias en pacientes internados en un hospital pediátrico de alta complejidad y su sistema de prevención, Rev Chil Nutr 2011; 38(1)30-9.

14. Vega-Briceño L. Contreras I. Prado F. Méndez M. Sánchez I. Hospital Josefina Martínez: Una breve reseña histórica, Neumol Pediatr. 2007; 2(1):3-5.

15. Barja S. Aspectos nutricionales en enfermedades respiratorias crónicas del niño, Neumol Pediatr. 2007; 2(1) 11-4.

16. Barja S. Enfermedades neurológicas en niños: Un adecuado apoyo nutricional, Neumol Pediatr. 2011; 6 (2): 61-6.

17. Barja S. Capo E. Briceño L. Jakubson L. Méndez M. Becker A. Anemia y déficit de hierro en niños con enfermedades respiratorias crónicas, Nutr Hosp. 2013; 28(3):787-93.

18. Jiménez R. Novo L. Santana S. Piñeiro E. Pérez E. Domínguez R. Evolución de la desnutrición hospitalaria, Rev Cub Pediatr. 2014; 86(3):298-307.

19. Morenos J. Oliveros L. Pedrón C. Desnutrición hospitalaria en niños, Acta Pediatr Esp 2005; 63:63-9.

20. Fuchs $G$. Gutiérrez N. Situación nutricional en pacientes pediátricos internados en un hospital pediátrico de Ciudad México, Rev Bras Nutr Clin. 2008; 23(3):178-83.

21. Reglamento Sanitario de los Alimentos. DTO. $N^{\circ} 977 / 96$
(D.OF. 13.05.97), 2015, 85-6.

22. FAO/OMS. Enterobacter sakazakii y otros microorganismos en los preparados en polvo para lactantes: informe de la reunión. Serie de evaluación de riesgos microbiológicos 6 . OMS, Ginebra, 2-5 de febrero de 2004 (acceso: http:// www.who.int/foodsafety/publications/micro/es_sp.pdf, Última visita 02/06/15) (2004).

23. FAO/WHO. Enterobacter sakazakii and Salmonella in powdered infant formula: meeting report, Microbiological Risk Assessment Series 10. (2006)

24. Iversen C. Mullane N. Mc Cardell B. Tall B. Lehner A. Fanning S. Stephan R. Joosten H. Cronobacter gen. nov., a new genus to accommodate the biogroups of Enterobacter sakazakii, and proposal of Cronobacter sakazakii gen. nov. comb. nov., C. malonaticus sp. nov., C. turicensis sp. nov., C. muytjensii sp. nov., C. dublinensis sp. nov., Cronobacter genomospecies 1, and of three subspecies, C. dublinensis sp. nov. subsp. dublinensis subsp. nov., C. dublinensis sp. nov. subsp. lausannensis subsp. nov., and C. dublinensis sp. nov. subsp. lactaridi subsp. nov., Int J Syst Evol Microbiol. 2008; 58:1442-7.

25. Saéz M. Llanos S. Tamayo R. Primer aislamiento de Cronobacter spp (Enterobacter sakazakii) en fórmula láctea en polvo producida en Chile, Rev Ch Salud Púb. 2012; 16(1):11-5.

26. Fang T. Gurtler JB. Huang L. Growth kinetics and model comparison of Cronobacter sakazakii in reconstituted powdered infant formula, J Food Sci. 2012; 77 (9): 247-55.

27. Parra J. Oliveras L. Rodriguez A. Riffo F. Jackson E. Forsythe S. Riesgo de contaminación por Cronobacter Sakazakii en leches en polvo para la nutrición de lactantes, Rev Chil Nutr. 2015; 42 (1): 83-9.

28. Coburn B. Grassl A. Finlay B. Salmonella, the host and disease: a brief review, Immunol Cell Biol. 2007; 85: 112-8.

29. CODEX ALIMENTARIUS. Código de prácticas de higiene para los preparados en polvo para lactantes y niños pequeños. CAC/RCP 66. 2008.

30. European Commission. Health \& Consumer Protection Directorate-General. Guidance document on the implementation of procedures based on the HACCP, and on the facilitation of the implementation of the HACCP in certain food business. Brussels, 16 November 2005, 4-13. (2005)

31. European Union. Commission Regulation (EC) No. 1441/2007 amending Regulation (EC) No. 2073/2005 on microbiological criteria for foodstuffs. Off J Eur Union. 2007; L 322, 12-29. (2007)

32. Gurtler JB. Kornacki J. Beuchat, L. Enterobacter sakazakii: A coliform of increased concern to infant health, Int J Food Microbiol. 2005; 104:1-34.

33. OMS. Preparación, almacenamiento y manipulación en condiciones higiénicas de preparaciones en polvo para lactantes - Directrices. "OMS en colaboración con la Organización de las Naciones Unidas para la Agricultura y la Alimentación". Suiza, 2007, 2-14. (2007). 